\title{
A Patient Monitoring System Using Internet of Things Technology
}

\author{
Ajaegbu Chigozirim ${ }^{*}$, Nwaocha O. Vivian ${ }^{2}$, Nzenwata J. Uchenna ${ }^{1}$, Adesegun A. Oreoluwa ${ }^{1}$ \\ ${ }^{1}$ Department of Computing and Engineering Sciences, Babcock University, Ogun State, Ilishan-Remo 121103, Nigeria \\ ${ }^{2}$ Department of Computer Science and Information Technology, National Open University Abuja, Jabi 900211, Nigeria
}

Corresponding Author Email: ajaegbuc@babcock.edu.ng

https://doi.org/10.18280/isi.250309

Received: 11 March 2020

Accepted: 7 May 2020

\section{Keywords:}

microcontroller, patient monitoring, internet of things, interfacing, internet

\begin{abstract}
Monitoring of patients have become one of the major problems in our society, particularly in large communities where the patient-to-doctor ratio is very small. This has resulted in the growing usage of remote monitoring systems around the world using various technologies like 'Internet of Things'(IoTs). The significant problem is that it is impossible for physicians to monitor all patients at once particularly in hospitals and clinics where there are clearly not many available. The health of patients would be compromised if they are not managed on time and they risk developing further complications. This research aims to develop a patient monitoring system, that incorporates the use of 'Internet of Things' technology to enable the device provide information about the patient's vitals over the internet. To achieve this, a system was developed using ATMEA328P microcontroller along with ESP8266 Wi-Fi module that enabled internet connectivity and was also interfaced with the microcontroller serially for exchange of data. Hence, a health monitoring system that renders information about the vitals of a recuperating patient, through the use of IoTs was developed. Furthermore, this study would enable decrease of manual checking thus, increasing the efficiency and throughput of medical professionals. The proposed system recorded a success rate of 80 percent of the transmitted data over the internet when tested on 10 individuals with accurate conversion of raw data into readable format in all successful transmitted data. The device would be of use to various people at home, hospital and other places.
\end{abstract}

\section{INTRODUCTION}

The advancement in medical technology demands optimum utilization of the automated systems. This entails upgrade of medical facilities generally to accommodate the changing technology in the medical world. Hitherto, measurement of blood pressure, glucose level, heart rate and other vitals have been attributed more to critically ill aged patients and most times come with delay. This sometimes could be inaccurate and lead to poor decision-making [1]. However, the advent of Internet of things has been progressing significantly as of late in the healthcare sector.

The concept of Internet of Things has been present for nearly four decades but only recently has it started to gain popularity and incorporation into the everyday aspects of human life. IoTs is expected to grow rapidly in the coming years and this convergence will unleash new dimension of services that will boost customer quality of life and business profitability, exploiting an opportunity known as 'connected life'.

Internet of Things innovation in the medical sector is generally linked to interconnection of accessible medical assets and provision of dependable and solid healthcare services with immediate decision making. The introduction of internet of things in medical services will continue to prompt drastic increase in productivity, profitability and quick analysis of medical data.

It is evident that the cost of medical care service is increasing rapidly in the society and thus emphasis to minimise and save cost for any healthcare industry. As indicated in the National Broadband Plan (2015) by the Federal Communications Commission (FCC), the utilization of remote patient checking under IoTs innovation will spare the healthcare industry $\$ 700$ billion for more than 15 to 20 years. Also, China spent over 50 million Yuan on healthcare infrastructure in 2001, amounting to $4.58 \%$ of its total GDP, with further increase to over 400 million Yuan in 2015, amounting to approximately $6.05 \%$ of the total GDP [2]. Hence, it is imperative to direct all focus to IoTs technology in medical sector in order to improve management of medical resources while delivering efficient and effective healthcare services [3].

In light of the increasing world's population, consistent healthcare and traditional monitoring of patients seem challenging. Thus, it is deemed necessary that a proper innovative monitoring system be put in place in the healthcare industry thus, the need for an IoTs monitoring system in the medical sector. Studies over the last four years, have demonstrated the abilities of IoTs in various aspects of patients monitoring such as the use of IoTs based patient monitoring system for body temperature, respiratory rate, heart beat and body movement using Raspberry Pi board [4], IoTs based patient monitoring and diagnostic prediction tool for stroke patients in order to control future occurrence [5] and also, wearable sensors have been used to demonstrate the efficiency of monitoring in real time sensing and analysis of various vital parameters of patients [6].

Hence, this study proposed and developed a monitoring 
system that should inform medical practitioners the vitals of a recuperating patient through the use of Internet of Things (IoTs) technology. This will allow for flexibility and portability in the medical sector while improving decision making and increasing the value of the medical sector.

\section{REVIEW OF RELATED WORKS}

Soumya and Kumar [7] looked at healthcare monitoring using Internet of Things and explained that everyday use of devices has given rise to employing health care with the help of smart techniques. They defined IoTs as a smart system consisting of a different type of sensor and has a real-world device connected through the use of the Internet via a wired or wireless network structure. They established that the cause of most deaths in India are diseases that are not monitored properly and that while some of these are curable, they can be completely prevented if the problem is identified immediately. Their aim was to develop a healthcare monitoring system that can keep track of a patient and have their vitals being monitored. They employed the use of a smart hospital system or SHS which is a two method phenomena; it first keeps track of a patient by automatic identification and biomedical device in the hospital, the second is by patient monitoring. The study ended with successfully recording and monitoring their participants' vitals for the duration of the testing period.

Jeyabalan [8] explained the applications of Internet of Things in Healthcare. Some of such categories or classifications are in critical care, follow-up care, and preemptive care, monitoring, diagnosing and treating. An issue raised is that the application of IoTs techniques varies from patient to patient as monitoring different patients can yield different results in terms of biotest sample tracking. Their aim was to give a general perspective on IoTs with relevant case studies, they attached an IoTs sensor to the wrists of select participants for a certain period of time. The devices will constantly emit messages to a base station about the location of the participants. At the start of testing, some data returned were not in a human readable format but after reconfiguring they were able to test accurately.

Ray [9] studied Home Health Hub Internet of Things and discussed how everything and everyday things are gradually being digitized as well as connected through the internet. The author stated that this huge advent has crept its way into one of the most cherished things about humans which is health. They highlighted an issue that IoTs research has been somewhat neglected due to heterogeneity and interoperability issues, they aimed to use the study to present or put forward a novel architectural framework for monitoring the health of elderly people at home.

In a study by Albakr et al. [10] on A Cloud-Assisted IoTs Framework, the authors explained that recent advances in cloud computing and Internet of Things have made their way into the healthcare industry. Their study noted that the integration of IoTs and cloud computing in the healthcare domain poses several technical challenges with less attention in the research community. Hence, authors proposed a smart city framework to help reduce the effect of the claimed challenges.

Xiang et al. [11] studied techniques that can be used to help improve IoTs technology as a whole. They highlighted that a strain on modern monitoring or on modern healthcare systems is being caused by a very large number of the aging population as well as a rise in the number of chronic illnesses we see today. Their aim was to propose a standard IoTs model that can be applied to the future of Healthcare systems. The model provided a comprehensive survey of some of the state-of-theart-technologies that are available right now with emphasis on sensors that can be used in monitoring various health attributes as well as short or long ranged communication standards and cloud technologies.

Banka et al. [12] proposed a Smart Healthcare Monitoring device that makes use of IoTs. They established that IoTs will be a key player in providing better medical care as well as facilities not only to patients but doctors as well. The aim of their study was to propose a system that consists of various medical devices that can communicate over a network and help to eliminate the issue of not being able to provide good healthcare to people in remote areas who have no access to hospitals by using a wearable device to do such tasks. The study was concluded with a wearable device kit using a microcontroller that would be able to record a patient's heart rate and blood pressure.

Kumar and Rajasekaran [4] opined that the significant challenges in the implementation of Internet of Things for healthcare applications is monitoring of patients from various locations. Hence, the authors proposed an IoTs based monitoring system capable of monitoring patients' body temperature, respiratory rate, heart beat and body movement using Raspberry Pi board.

Ani et al. [5] proposed a patient monitoring system for stroke-affected patients in order to minimize future recurrence of same through the process of alarming the doctor and caretaker on variation in risk factors of stroke disease. This was achieved using classification algorithms for the diagnosis and prediction.

Verma and Sood [13] used the concept of fog computing at the smart gateway to propose a remote patient health monitoring in smart homes. Authors employed embedded data mining, distributed storage and notification services at the edge of the network, where event triggering-based data transmission methodology was adopted in order to process the patient's real-time data at fog layer.

Archip et al. [14] looked at the steps taken to design and build a low-cost modular monitoring system prototype, with the aim of offering mobile support in order to facilitate faster and better medical interventions during emergency cases. Authors made use of low-power dedicated sensor arrays for EKG, SpO2, temperature and movement for the system design.

Rahman et al. [15] developed an intelligent patient monitoring system that automatically screens patients' health conditions using various sensors and also extracts the bio signal, ECG using an ECG sensor. Authors noted that, their data was processed using Raspberry Pi while copy of information was kept on IoTs cloud.

Gomez and Oviedo [16] developed a solution based on an ontology with ability to monitor the health status and offer recommendations of workout routines with chronic diseases architecture.

Mekki et al. [17] proposed an IoTs health monitoring architecture by allowing distributed security strategy. The authors further demonstrated the interaction between the system and other actors, where the major security requirements in the applied Wireless Body Sensor Network (WBSN) were explored.

Jiang et al. [18] proposed a wireless biopotentials remote monitoring and processing system using a case study of facial 
expression recognition. The study implemented the IoTs monitoring system on the basis of an eight channel biopotential measurement device, Wi-Fi module as well as signal processing and classification.

Li et al. [19] proposed an IoTs pervasive monitoring system with data acquisition part and data transmission part, with the ability to send patients' physical signs to remote medical application in real time.

From the review, it was observed that one major challenge apart from securing of patient data is that at the time of conversion of raw data into the dashboard on the internet, most of the existing systems returned unreadable data strings. Hence the need for a more robust system that could minimize the rate of such error.

\section{METHODOLOGY}

The microcontroller which is an ATMEGA328P is the heart of the system. The ESP8266 Wi-Fi module, employed to connect to the internet was interfaced with the microcontroller serially for exchange of data between themselves.

The heart rate sensor and temperature sensor used was to measure the heartbeat and temperature of the user respectively. This was connected to the microcontroller and was also used to send raw data to the microcontroller. The microcontroller would then translate that raw data into a readable string (format) and display it on the output device (Liquid crystal display).

The output would also display on the ThingSpeak platform over the Internet. The platform was interfaced with the device with an API key which would be platform specific. This would also make it unique per device. Figure 1 and 2 show the block and schematic diagram of the proposed system respectively.

The hardware and Design tools used to construct the device were:
a) AT Mega 328p Microprocessor
b) ESP8266 Wi-Fi module
c) Pulse sensor
d) LM35 Temperature sensor
e) LCD Display $(16 \times 2)$
f) Resistors
g) Crystal Oscillator
h) LED
i) Diode
j) Breadboard
k) SIM800 GSM Module
1) Connecting Wires
m) Capacitors
n) Battery Charger

\section{SYSTEM ANALYSIS}

\subsection{Power supply unit}

ATmega-328 is essentially an Advanced Virtual RISC (AVR) micro-controller. It supports data up to eight (8) bits. ATmega-328 has $32 \mathrm{~KB}$ internally built-in memory. It consists of an in-built EEPROM memory of $1 \mathrm{~KB}$ and a SRAM memory is of $2 \mathrm{~KB}$. It has $8 \mathrm{Pin}$ for ADC operations, which all combines to form Port A (PA0 - PA7). It also has 3 built-in Timers, two of them are 8 Bit timers while the third one is 16Bit Timer, an Arduino UNO is required to use the microcontroller as it is centred on the atmega328 Microcontroller. It operates between the ranges of $3.3 \mathrm{~V}$ to $5.5 \mathrm{~V}$ but $5 \mathrm{~V}$ is typically used as the standard. Its brilliant features include the cost effectiveness, low power dissipation, programming lock for safety purposes, and real timer counter with separate oscillator. See Figure 3.

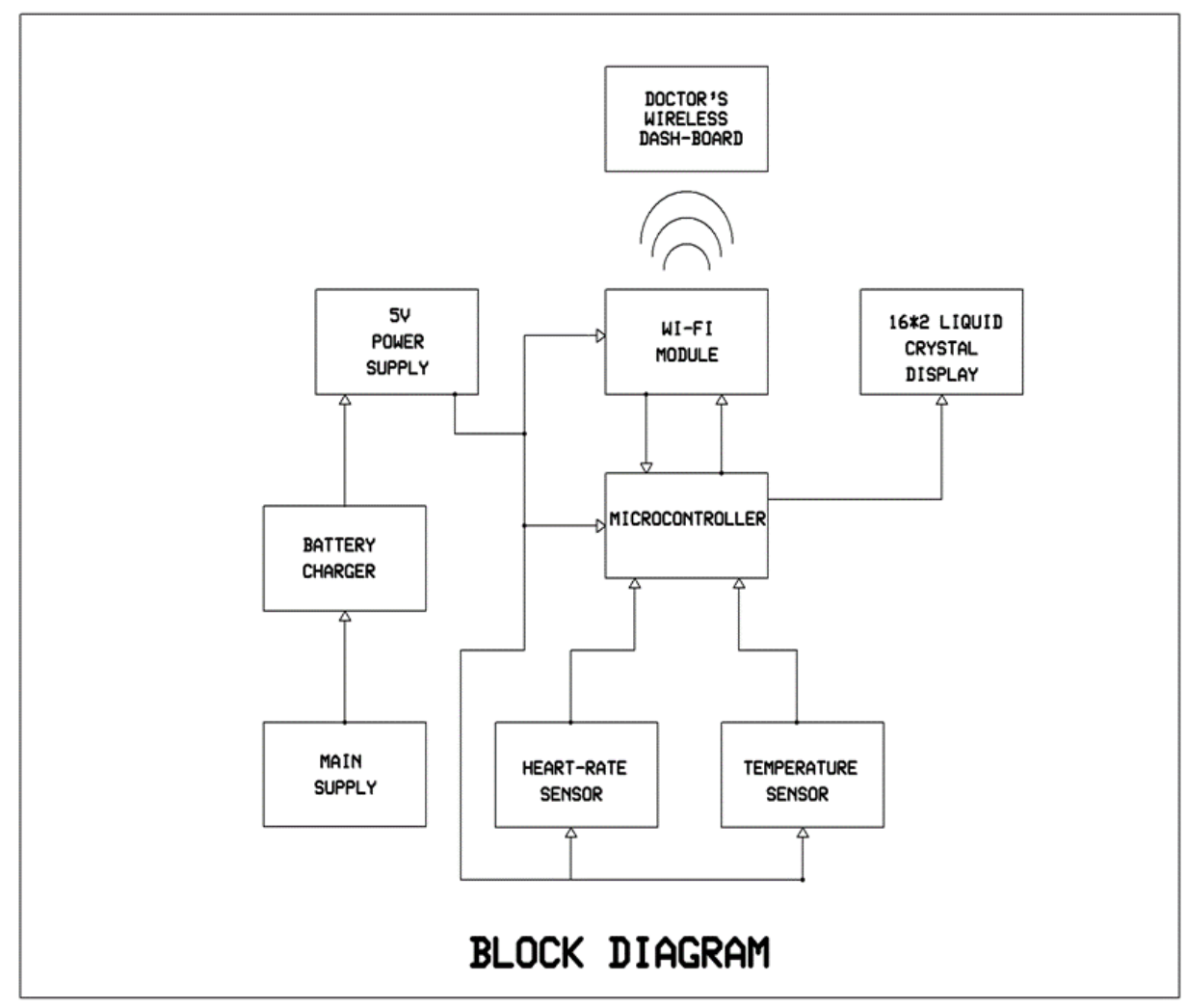

Figure 1. IoTs patient monitoring system block diagram (Source: Authors, 2020) 


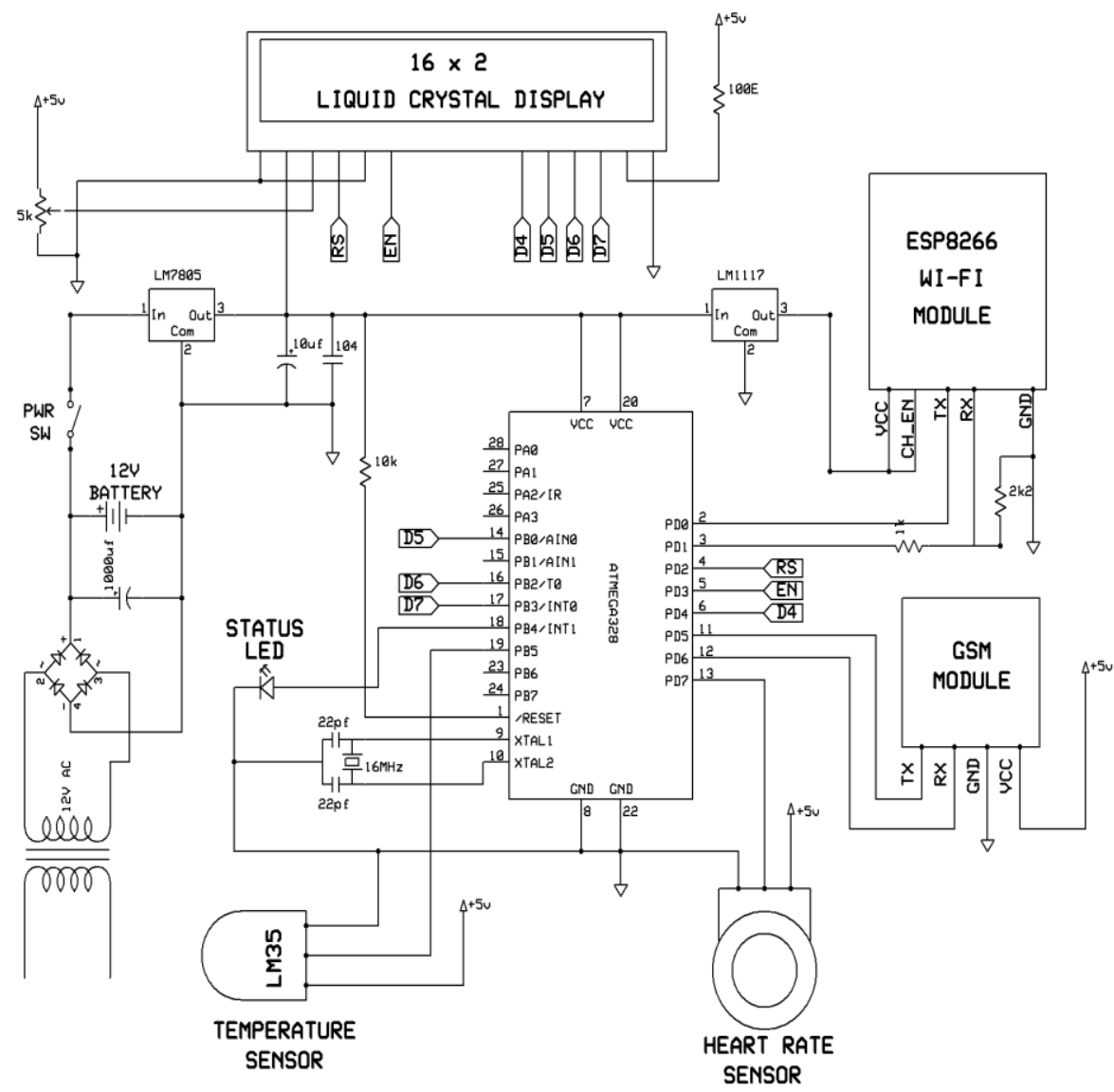

Figure 2. IoTs patient monitoring system (Source: Authors, 2020)

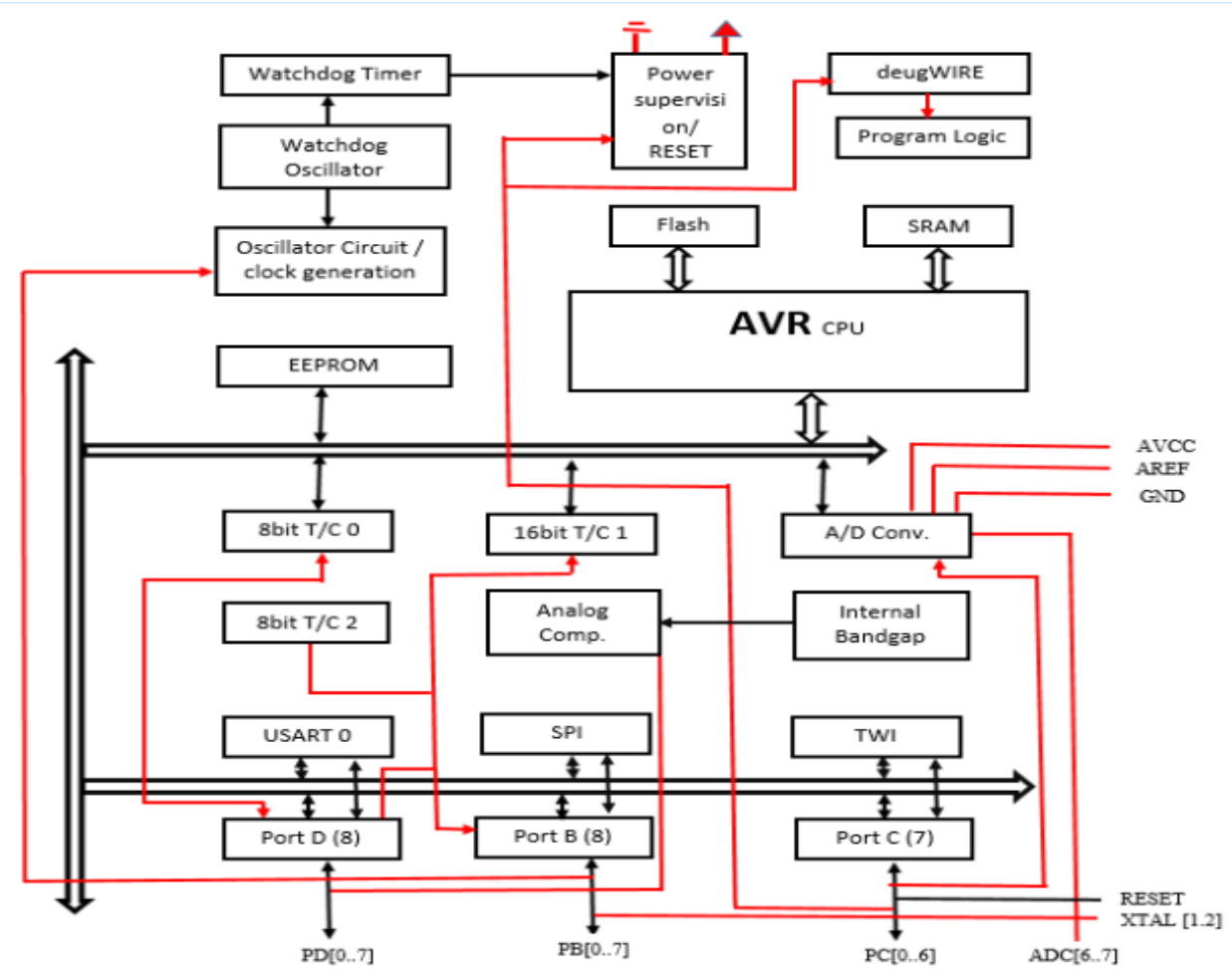

Figure 3. ATMEGA 328P Architecture (Source: elprocus.com, n.d)

\subsection{System requirements}

For the IoTs Patient Monitoring System to function as required, there are certain prerequisites that have to be met.
These necessities incorporate the following:

- The system should incorporate a microcontroller.

- The system must be able to operate on a battery pack. 
- The system ought to have adequate protection in the form of a case to protect against atmospheric conditions such as rain, snow etc.

- The temperature and pulse-rate sensor must be properly configured and attached to ensure that the results are optimal.

\subsection{Functional requirement}

This reflects functions that are meant to be given by the system and the performance that the system is intended to produce by providing a certain data. The functional requirements help to determine what a system developer has to construct in order to have a solution. The functional requirement of this device are as follows:

- Measurement utility

- Display utility

\subsection{Operation of patient monitoring system}

The construction of the IoTs Patient Monitoring System is done following the processes explained below:

The system requires a 5V DC power supply to operate. To achieve the $5 \mathrm{~V}$ required to supply the microcontroller and other components on the circuit, there is need to reduce the $9 \mathrm{~V}$ readily designed to $5 \mathrm{~V}$ and this is implemented with the usage of a voltage regulator IC. The IC used in this case is the LM7805 regulator IC. The first two numbers of the IC denote that it is a positive voltage supply IC while the last one denotes the output supply voltage. There is also the need of a battery charger for the system to work when there is no DC supply.

The microcontroller which is the heart of the system would be interconnected with the Wi-Fi module serially i.e. the microcontroller and the $\mathrm{Wi}-\mathrm{Fi}$ module would be able to communicate interchangeably. A host device will allow the Wi-Fi module to gain internet access which in return will allow the $\mathrm{Wi}-\mathrm{Fi}$ module to tell the microcontroller it is connected to the internet.

The heart rate sensor and temperature sensor that would be used to measure the heartbeat and temperature of the user respectively would also be connected to the microcontroller and would send raw data to the microcontroller which would translate the raw data into a readable string (format) and display it on the output device (Liquid crystal display).

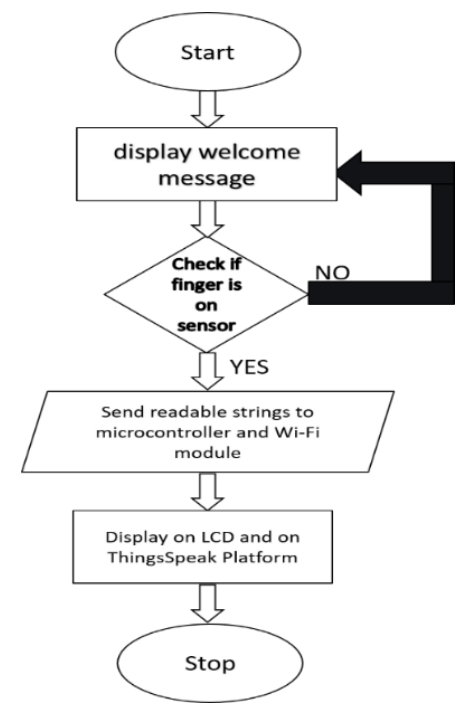

Figure 4. System flow chart (Source: Authors, 2020)
The output would also display on the Thingspeak platform over the Internet. The platform will be interfaced with the device with an API key which would be platform specific (see Figure 4 for system flow chart of the proposed algorithm for enabling interfacing). Hence, making it unique per device.

\subsection{Proposed system algorithm}

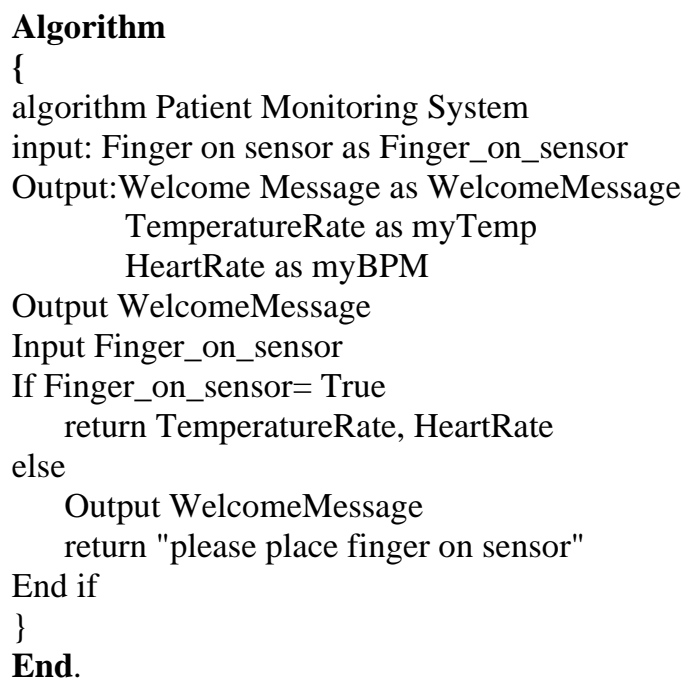

From the above algorithm, the user would first have to turn on the device, a message should be displayed indicating that the device is working. Once the device is turned on, it would require an internet connection on the specified host device, if no internet access is detected an error message would be revealed on the LCD display. The user would then proceed to place their finger on either the temperature sensor or pulse rate sensor, after which a value is returned back to the LCD and the ThingSpeak dashboard.

\subsection{Design phase difficulties encountered with the system}

\subsubsection{WIFI module}

During the construction phase we originally intended to configure the device using an Arduino Shield or Heavy Module but the module being difficult to configure had to be replaced with an ESP8266 which is cost effective along with its software stacks.

\subsubsection{Network connectivity}

Network connectivity within the university environment was poor at times which made it difficult for the device to establish a secure connection to the patient dashboard. To solve this a WIFI router was procured.

\subsubsection{Resource depravation}

Due to time and financial constraints, some components that were originally intended to improve the efficiency of the system were no longer added.

\section{CONCLUSION}

The patient monitoring system is a step towards integrating IoTs technology into the modern health care system. The patient monitoring system is designed to provide a resolution to an existing problem in hospitals. Doctors and nurses will no longer have to move from bed to bed for check-ups. In addition, 
it would aid relatives and loved ones of these patients to regularly monitor them when out of the hospital. Hence, issues or complications that may arise can be swiftly addressed.

The proposed system is able to measure the temperature and heart rate and was able to synchronise the data unto an LCD display and the cloud. The proposed system had a success rate of 80 percent of the transmitted data over the internet when tested with 10 individuals with accurate conversion of raw data into readable format in all successful transmitted data. This device has been conceived to help in regularizing the health condition of most individuals by keeping track of their vitals and it also helps in connecting the individual to the doctor. However, its prospects and challenges are listed below:

\subsection{Prospects of the proposed system}

1. Patients do not need to stay in hospitals for prolonged periods of time due to remote patient monitoring.

2. The burden of routine check-ups performed by required personnel are largely minimized.

3. Patients who have some form of disability to an extent can benefit from the device due to regular hospital visits being greatly shortened.

4. Doctors would no longer require large amounts of manual data retention as data would be stored on the cloud rather than in large files.

5. Doctors would benefit greatly as they can easily make reference to a patients history if any sudden change occurs during monitoring.

\subsection{Challenges of the proposed system}

1. Unapproved access to centralization. This means that once an unauthorized individual gains access to the central server, information could be leaked.

2. The device does not retain data for long periods.

\subsection{Comparison with existing system}

Table 1. Comparison table between existing system and proposed system

\begin{tabular}{cccc}
\hline S/N & Parameters & $\begin{array}{c}\text { Existing } \\
\text { system }\end{array}$ & $\begin{array}{c}\text { Proposed } \\
\text { system }\end{array}$ \\
\hline 1 & Must be able to secure an internet \\
connection & Yes & Yes \\
2 & $\begin{array}{c}\text { Must be able to display parameters } \\
\text { accurately }\end{array}$ & Yes & Yes \\
3 & $\begin{array}{c}\text { Must be able to notify required } \\
\text { personnel in case of an emergency } \\
\text { Must be able to display data locally } \\
\text { and over a secure internet } \\
\text { connection }\end{array}$ & No & Yes \\
4 & $\begin{array}{c}\text { Yes } \\
\text { Must be able to secure the patients } \\
\text { data }\end{array}$ & No & Yes \\
6 & $\begin{array}{c}\text { Must be able to show the precise } \\
\text { location of a patient }\end{array}$ & No & No \\
\hline
\end{tabular}

\section{FUTURE WORKS}

The system can be expanded by improving on its access control to centralized server and also improving on its data retention level. Also, some capabilities and work rate could be incorporated for better productivity such as:

- Addition of glucose level sensor to the current system.
- The system can be optimized to send SMS messages to the doctor/guardian whenever there is a crisis.

- A web interface can be built from scratch to connect the hardware instead of using an already existing platform.

- A GPS module would help to improve precise patient monitoring such that the doctor would be able to find out the location of the patient in the case of a severe emergency.

Hence, this device can be applied to various areas of the society and is specially recommended for people who are in need of constant care and check-up such as: Patients in hospitals, convalescents, the elderly and the disabled.

\section{REFERENCES}

[1] Gardner, R.M., Shabot, M.M. (2013). PatientMonitoring System. https://collections.lib.utah.edu/dl_files/f0/a3/f0a3781fc9 48833aa480ad7085b49dc200e78de6.pdf.

[2] Wan, J., Al-awlaqi, M.A.A.H., Li, M., O'Grady, M., Gu, X., Wang, J., Cao, N. (2018). Wearable IoT enabled realtime health monitoring system. EURASIP Journal on Wireless Communications and Networking, 298(1). https://doi.org/10.1186/s13638-018-1308-x

[3] Ibrahim, A.A., Wang, Z.P. (2018). IoT patient health monitoring system. Journal of Engineering Research and Applications, 8(1): 77-80. https://doi.org/10.9790/96220801037780

[4] Kumar, R., Rajasekaran, M.P. (2016). An IoT based patient monitoring system using raspberry Pi. 2016 International Conference on Computing Technologies and Intelligent Data Engineering (ICCTIDE'16), Kovilpatti, https://doi.org/10.1109/ICCTIDE.2016.7725378

India.

[5] Ani, R., Krishna, S., Anju, N., Aslam, M.S., Deepa, O.S. (2017). IoT based patient monitoring and diagnostic prediction tool using ensemble classifier. 2017 International Conference on Advances in Computing, Communications and Informatics (ICACCI), Udupi, India. https://doi.org/10.1109/ICACCI.2017.8126068

[6] Vippalapalli, V., Ananthula, S. (2016). Internet of things (IoT) based smart health care system. 2016 International Conference on Signal Processing, Communication, Power and Embedded System (SCOPES), Paralakhemundi, India. https://doi.org/10.1109/SCOPES.2016.7955637

[7] Soumya, S., Kumar, S. (2019). Healthcare Monitoring Using Internet of Things. First International Conference on Artificial Intelligence and Cognitive Computing. Advances in Intelligent Systems and Computing, Springer, Singapore. https://doi.org/10.1007/978-98113-1580-0_47

[8] Jeyabalan, K. (2016). Home Healthcare and Remote Patient Monitoring. Internet of Things and Data Analytics https://doi.org/10.1002/9781119173601.ch41

[9] Ray, P.P. (2014). Home Health Hub Internet of Things (H3IoT): An architectural framework for monitoring health of elderly people. 2014 International Conference on Science Engineering and Management Research (ICSEMR), Chennai, India. https://doi.org/10.1109/ICSEMR.2014.7043542 
[10] Albakr, H.S., Hassan, M.M., Al-Dossari, H. (2014). A cloud-assisted internet of things framework for pervasive healthcare in smart city environment. Proceedings of the 1st International Workshop on Emerging Multimedia Applications and Services for Smart Cities, pp. 9-13. https://doi.org/10.1145/2661704.2661707

[11] Xiang, W., Atkinson, I.M., Baker, S.B. (2017). Internet of things for smart healthcare: Technologies, challenges, and opportunities. IEEE Access, 5: 26521-26544. https://doi.org/10.1109/ACCESS.2017.2775180

[12] Banka, S., Madan, I., Saranya, S. (2018). Smart Healthcare Monitoring using IoT. International Journal of Applied Engineering Research, 13(15): 11984-11989.

[13] Verma, P., Sood, S.K. (2018). Fog assisted-Iot enabled patient health monitoring in smart homes. IEEE Internet of Things Journal, 5(3): 1789-1796. https://doi.org/10.1109/JIOT.2018.2803201

[14] Archip, A., Botezatu, N., Serban, E., Herghelegiu Pand Zala, A. (2016). An IoT based system for remote patient monitoring. 2016 17th International Carpathian Control Conference (ICCC), Tatranska Lomnica, Slovakia. https://doi.org/10.1109/CarpathianCC.2016.7501056

[15] Rahman, A., Rahman, T., Ghani, N.H., Hossain, S., Uddin, J. (2019). IoT based monitoring system using
ECG sensor. 2019 International Conference on Robotics, Electrical and Signal Processing Techniques (ICREST), Dhaka, Bangladesh, Bangladesh. https://doi.org/10.1109/ICREST.2019.8644065

[16] Gomez, J., Oviedo, B.(2016). Patient monitoring system based on internet of things. Procedia Computer Science, 83: 90-97. https://doi.org/10.1016/j.procs.2016.04.103

[17] Mekki, N., Hamdi, M., Aguili, T., Kim, T. (2017). Scenario-based vulnerability analysis in iot-based patient monitoring system. In Proceedings of the 14th International Joint Conference on e-Business and Telecommunications (ICETE 2017) - Vol. 4: SECRYPT, Madrid, Spain, pp. 554-559. https://doi.org/10.5220/0006473005540559

[18] Jiang, M., Gia, T.N., Anzanpour, A., Rahmani, A., Westerlund, T., Salantera, S., Liljeberg, P., Tenhunen, H. (2016). IoT-based remote facial expression monitoring system with sEMG signal. 2016 IEEE Sensors Applications Symposium (SAS), Catania, Italy. https://doi.org/10.1109/SAS.2016.7479847

[19] Li, C., Hu, X., Zhang, L. (2017). Pervasive healthcare; Internet of Things; heart diease; monitoring system. Procedia Computer Science, 112: 2328-2334. https://doi.org/10.1016/j.procs.2017.08.265 